\title{
Itinéraires Itinéraires
}

Littérature, textes, cultures

2012-1 | 2012

Genres et avant-gardes

\section{L'allégorie de la femme-enfant alias Gisèle Prassinos comme aporie de genre dans le surréalisme}

Annie Richard

\section{(2) OpenEdition}

Journals

Édition électronique

URL : http://journals.openedition.org/itineraires/1314

DOI : $10.4000 /$ itineraires. 1314

ISSN : 2427-920X

Éditeur

Pléiade

Édition imprimée

Date de publication : 1 septembre 2012

Pagination : 147-159

ISBN : 978-2-296-55776-5

ISSN : 2100-1340

Référence électronique

Annie Richard, «L'allégorie de la femme-enfant alias Gisèle Prassinos comme aporie de genre dans le surréalisme », Itinéraires [En ligne], 2012-1 | 2012, mis en ligne le 01 septembre 2012, consulté le 30 avril 2019. URL : http://journals.openedition.org/itineraires/1314; DOI : 10.4000/itineraires.1314

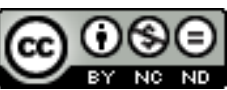

Itinéraires est mis à disposition selon les termes de la licence Creative Commons Attribution - Pas d'Utilisation Commerciale - Pas de Modification 4.0 International. 


\title{
L'allégorie de la femme-enfant alias Gisèle Prassinos comme aporie de genre dans le surréalisme
}

\begin{abstract}
The organic link between automatic writing, surrealism's subversive objective and the femme-enfant figure, produces a contradiction revealed by Man Ray's photograph of the 1934 encounter between André Breton's group and "Alice II," as they named the young poetess Gisèle Prassinos. Such a mythological appellation, although proceeding from the modern objective approach to the world, in no way disrupts the traditional analogic structure of gendered identities and prevents the "Sublime Point" from evolving beyond the masculine/feminine opposition at the basis of binary thought.
\end{abstract}

Keywords : child-woman, sublime point, automatic writing, Man Ray, Gisèle Prassinos Mots clés : femme-enfant, point sublime, écriture automatique, Man Ray, Gisèle Prassinos

«C'est la révolution permanente en belles images coloriées à un sou - elles n'existent plus - mais le ton de Gisèle Prassinos est unique : tous les poètes en sont jaloux ${ }^{1}$. » André Breton intronise ainsi Gisèle Prassinos dans la première édition de l'Anthologie de l'humour noir où elle est la seule auteure. La deuxième femme, figurant dans la deuxième édition, est Leonora Carrington. Cette élection touche éminemment au genre : pour André Breton, Gisèle Prassinos et Leonora Carrington sont retenues explicitement, dès la phrase introductrice de chaque notice, en tant que figures spécifiquement féminines, celle de la femme-enfant pour Gisèle Prassinos - « Il sied encore de dresser sur l'horizon de l'humour noir ce que Dali a appelé le "monument impérial à la femme-enfant" »-, et celle de la sorcière pour Leonora Carrington - « Michelet, qui a rendu si belle justice à la Sorcière, met chez elle en lumière ces deux dons, inestimables du fait

1. André Breton, Anthologie de l'humour noir [1940; 1950; 1966], repris dans Euvres complètes, t. II, Paris, Gallimard, coll. « Bibliothèque de la Pléiade », 1992, p. 1168. 
qu'ils ne sont départis qu'à la femme, "l'illuminisme de la folie lucide" et "la sublime puissance de la conception solitaire" »".

Ces figures sont placées sous l'égide de grandes voix masculines : celles de Michelet, de Dali, convoquées par celle d'André Breton ${ }^{3}$. Le lecteur est en effet incité à les mettre sur un même plan : le rapprochement entre la femme-enfant et la sorcière est favorisé par André Breton lui-même quand il adjoint dans la deuxième édition de l'Anthologie de l'humour noir, la sorcière à la femme-enfant ${ }^{4}$, comme quand plus tard dans Arcane 17 (1944) s'opère un glissement de la femme-enfant vers Mélusine :

\begin{abstract}
dans Arcane 17, Breton affirme catégoriquement que «sous l'écroulement des cheveux dédorés (de Mélusine) » se compose le type (« tous les traits distinctifs ») de la femme-enfant, " de cette variété si particulière qui a toujours subjugué les poètes, parce que sur elle le temps n'a pas de prise » (souligné par A.B.) ${ }^{5}$.
\end{abstract}

Cette similitude apparente masque une dissymétrie notoire : la femmeenfant ne puise pas dans l'imaginaire traditionnel mais est une création, un mythe moderne, nouveau «mythologème ${ }^{6}{ }$ » investi de façon continue par André Breton, d'une portée révolutionnaire depuis la figuration ${ }^{7}$ de l'écriture automatique sous les traits d'une « écolière ambiguë8 ${ }^{8}$ ", innocemment perverse. S'interrogeant sur sa genèse, Gérard Legrand évoque, à titre d'hypothèses - car il dit ignorer vraiment « l'origine de la formule femmeenfant» au sein du surréalisme -, des sources littéraires (Bettina Von Arnim ou le goût des romantiques allemands pour les très jeunes femmes) et biographiques (la pensée de Breton pour sa fille Aube dont il est séparé au moment de l'écriture d'Arcane 17) 9 . Or ce «mythologème », central dans la radicalité de la révolution surréaliste puisqu'il est étroitement lié à la pratique de l'écriture automatique, perdure et s'amplifie dans le discours d'André Breton : le type de la femme-enfant évolue des «mineures ${ }^{10}$ »

2. Ibid., respectivement p. 1167 et p. 1162 .

3. Georgiana Colvile a étudié les effigies, fictions de femmes qui constituent «le mythe de la femme, érigé au centre du corpus surréaliste » (Georgiana Colvile, Scandaleusement d'Elles, Paris, Jean-Michel Place, 1998, p. 9). Voir également, du même auteur : " Filles d'Hélène, sœurs d'Alice », dans Jacqueline Chénieux-Gendron et Yves Vadé (dir.), Pensée mythique et surréalisme, Paris, Lachenal et Ritter, coll. « Pleine Marge », 1996, p. 245-262, et « Les femmes-fantômes du surréalisme », Emmnanuel Rubio (dir.), L'Entrée en surréalisme, Paris, Phénix Éditions, 2004, p. 155-171.

4. La première édition ne retenait que Gisèle Prassinos.

5. Gérard Legrand, «À propos de la Femme-enfant : Contribution à une typologie de la femme surréaliste », Obliques, nº 14-15, 1977, p. 11.

6. Ibid., p. 9.

7. Couverture de La Révolution surréaliste, $\mathrm{n}^{\circ}$ 9-10, octobre 1927.

8. André Breton, Anthologie de l'humour noir, op. cit., p. 1167.

9. Gérard Legrand, op. cit., p. 10-11.

10. Ibid., p. 11. 
contemporaines de l'écriture du Second manifeste, qui évoquent plutôt une enfant-femme, à l'exaltation dans la femme de sa part intrinsèquement « enfantine », la femme-enfant d'Arcane 17 dont la destinatrice est Élisa.

La problématique du genre permet d'interroger de manière aussi radicale cette articulation entre le genre majeur pour les surréalistes, débordant tous les genres et la littérature même pour déboucher sur la transformation de la vie, le genre poétique, et son expression genrée, la femme-enfant. Autrement dit, que révèle ce « mythologème » sur la visée épistémologique du surréalisme, définie dans le Second manifeste de $1930^{11}$ comme «l'espoir de détermination » $\mathrm{d}^{\prime}$ ' u un certain point de l'esprit d'où la vie et la mort, le réel et l'imaginaire, le passé et le futur, le communicable et l'incommunicable, le haut et le bas cessent d'être perçus contradictoirement $»^{12}$ ?

La première révélation est de l'ordre de l'évidence : le couple manquant des « vieilles antinomies » à briser pour accomplir l' « envolée de l'esprit », et partant de la vie, est celui du genre. Il n'y pas de catégorie duelle féminin/masculin à dépasser malgré son lien avec la préoccupation essentielle de l'éros surréaliste. Certes celui-ci se situe sur le plan de la sexualité, orientation sexuelle intériorisée de l'ordre du désir, mais de même que la sexualité ne peut s'affranchir du genre qu'en s'y référant, c'est l'ordre même du désir qui sert « l'essor d'une pensée ${ }^{13}$ » chez André Breton: pourquoi donc l'unité à percevoir ne concernerait-elle pas le partage des sexes?

Alors qu'une déstabilisation du genre se produit dans la diversité de l'expression littéraire et artistique surréaliste ${ }^{14}$, le discours théorique n'interroge pas dans ce domaine les catégories de pensée : ainsi le mouvement dominant du dépassement des contraires dans l'Amour fou (1937) vise la fusion dans l'Un par le mythe de l'Androgyne qui laisse intacte la spécificité de la femme et le caractère essentiel de la différence des sexes.

Le paradoxe existe donc, d'autant plus troublant pour qui prend comme objet d'étude l'œuvre de l'artiste qui incarne la femme-enfant, Gisèle Prassinos, de 1934, année de sa découverte par les surréalistes, à 1939, date de sa dernière publication officiellement surréaliste, Sondue ${ }^{15}$. Ayant construit, en tant que commissaire de la rétrospective de la Bibliothèque historique de la

11. Voir le frontispice de l'édition de luxe avec l'inscription «femme-enfant» de la main de Dali sur sa gravure avec un torse masculin et des têtes masculines dont celle de Guillaume Tell.

12. André Breton, Second manifeste du surréalisme [1930], repris dans Euvres complètes, t. I, Paris, Gallimard, coll. « Bibliothèque de la Pléiade », 1988, p. 781.

13. André Breton, L'Amour fou [1937], repris dans Euvres complètes, t. II, op. cit., p. 750. 14. Analysée notamment par Dawn Ades, "Surrealism, male/female », dans Surrealism desire unbound, catalogue de l'exposition, Londres, Tate, 20 septembre 2001-1 ${ }^{\text {er }}$ janvier 2002, Londres, Tate Publishing, 2002.

15. Gisèle Prassinos, Sondue, Paris, GLM, coll. « Biens nouveaux », 1939. 
ville de Paris ${ }^{16}$, une vision globale de cette œuvre, le dépassement des sexes m'en apparaît comme un axe essentiel : les tentures en feutrine notamment, qu'elle confectionne de 1967 à 1988, remontent, en parodiant les grands sujets de la peinture d'Histoire, à la source religieuse d'une séparation sexuée absolue et hiérarchique ${ }^{17}$. Son humour libéré dans la confection de ces tableaux en tissu qu'elle considérait comme un jeu parvient de façon éclatante, dans sa version de Salomé et saint Jean-Baptiste, à une véritable vision du genre affranchi du sexe ${ }^{18}$.

Ainsi le «point sublime» genré, dirions-nous aujourd'hui, de la partition des sexes, avait été atteint spontanément, en somme automatiquement, par l'« Alice II » du Dictionnaire abrégé du surréalisme, alias Gisèle Prassinos ${ }^{19}$, parvenue à une maturité complètement ignorée de ses anciens thuriféraires. En effet quand le nom de Gisèle Prassinos réapparaît, en pleine lumière sur la scène littéraire parisienne, salué par la critique, avec le récit d'enfance autofictionnel Le Temps n'est rien (Plon, 1958), on aurait pu attendre de la part d'André Breton une simple mention, voire un rejet explicite condamnant la forme romanesque de ce retour ${ }^{20}$. Mais le silence total sur l'existence, en dépit du développement continu de son invention créatrice, de l'artiste adulte Gisèle Prassinos, restée femme-enfant, fait office d'effacement, entériné par l'histoire du surréalisme jusqu'à aujourd'hui.

La problématique du genre permet d'interroger autrement ce silence ou ces réticences : pourquoi le surréalisme en tant que mouvement collectif tient-il à occulter la maturité de son «Alice II » sinon pour lier, à la manière d'un manifeste, avec ce que cela comporte de grossissement polémique, son objectif téléologique révolutionnaire de bouleversement de la pensée humaine à un éternel féminin sur lequel le temps n'a pas de prise?

Le mode d' ' entrée en surréalisme ${ }^{21} »$ de Gisèle Prassinos, à savoir la photographie par Man Ray de sa première rencontre avec le groupe

16. L'exposition « Le Monde suspendu de Gisèle Prassinos » tirait son titre du livre qui l'a inspirée : Annie Richard, Le Monde suspendu de Gisèle Prassinos, Paris, H.B. Éditions, 1997. L'exposition eut lieu à la Bibliothèque historique de la ville de Paris du 13 mars au 3 mai 1998.

17. Annie Richard, La Bible surréaliste de Gisèle Prassinos, Wavre (Belgique), Mols, 2004. 18. Annie Richard, «Salomé ou les avatars de la femme-enfant», dans La Femme s'entête, Actes du colloque "La part du féminin dans le surréalisme » organisé par Georgiana Colvile et Katharine Conley (Centre culturel international de Cerisy-la-Salle, août 1997), Paris, Lachenal et Ritter, coll. « Pleine Marge », 1998.

19. Annie Richard, "L'Alice II de Breton et la quête du "point sublime" du partage des sexes dans La Bible surréaliste de Gisèle Prassinos ", dans Le Surréalisme en son temps et aujourd'hui, Actes du colloque international de la faculté de philologie de Belgrade du 21 au 23 septembre 2006, Belgrade, Faculté de philologie de Belgrade, 2007.

20. Annie Richard, «Le Grand Repas, roman surréaliste », Mélusine, n 16, " Cultures et contre-cultures », 1997, p. 353-363.

21. Voir Annie Richard, «L'entrée en surréalisme à l'épreuve de la photographie », dans L'Entrée en surréalisme, op. cit., p. 173-186. 
surréaliste en 1934, permet, pour répondre à cette question, de se placer sur le plan de la révolution surréaliste qui est celui d'une rupture épistémologique. Lire la photo de Gisèle Prassinos sous cet angle situe l'appropriation de cette figure d'identité sexuée, la femme-enfant, au niveau même de l'objectif avant-gardiste du surréalisme de transformation radicale de notre façon d'appréhender la réalité.

En effet la « fabrique des images ${ }^{22}$ », selon Philippe Descola, procède d'une vision du monde selon des choix de discernement intériorisés par notre culture. À partir de réalisations provenant de tous les continents, l'anthropologue établit une typologie dans l'espace et dans le temps des différentes réalités sous-tendues par les images.

Rappelons que la langue établit le lien entre voir et penser : le mot voir est de la même famille que le mot idée, tous deux issus de l'indo-européen weid qui donne idein en grec et idea chez Platon : forme idéale concevable par la pensée. L'étymologie de théorie est théâtre, issu d'un mot grec signifiant contempler : la théorie est spectacle, fête solennelle, défilé des députations des villes grecques à Delphes et à partir de Platon contemplation, spéculation de l'esprit. Les surréalistes ne désavoueraient sans doute pas ce recours à la mémoire de la langue cautionnée par Freud - « ce que Freud nous rappelle tout le temps », dit Lacan, c'est que, "pour retrouver la trace de l'expérience accumulée de la tradition, des générations, l'approfondissement linguistique est le véhicule le plus certain de la transmission d'une élaboration qui marque la réalité psychique ${ }^{23} »$.

Notre proposition est d'utiliser la perspective épistémologique développée par Philippe Descola dans La Fabrique des images ${ }^{24}$ pour repenser, en nous dégageant des textes représentatifs, l'ontologie avantgardiste affichée dont procède l'image de l'entrée en surréalisme de Gisèle Prassinos, ontologie explicite de rupture avec la pensée rationaliste traditionnelle portée par la figure de la femme-enfant. Gisèle Prassinos est définie en effet dans le mouvement par une photo célèbre de Man Ray qui accompagne la publication de sa première plaquette La Sauterelle

22. Voir l'exposition au musée du Quai Branly, Paris, du 16 février 2010 au 11 juillet 2011, sous la direction de Philippe Descola, et le catalogue La Fabrique des images, visions $d u$ monde et formes de la représentation, Paris, Somogy, 2010.

23. Jacques Lacan, Le Séminaire, L'éthique de la psychanalyse, livre VII, Paris, Seuil, 1986, p. 56.

24. Op. cit., note 22 : les visions du monde recensées par Philippe Descola sont en nombre restreint : il n'y a pas des possibilités infinies de discernement par les sens ou par l'imagination. Quatre sont prépondérantes, sans être totalement exclusives, selon de grandes zones géographiques : l'animisme, par exemple celui des Indiens du cercle polaire d'Amérique du Nord, le totémisme australien, l'analogisme, dominant en Europe de l'Antiquité à la Renaissance, dans les civilisations d'Orient et dans les communautés indiennes d'Amérique, enfin le naturalisme majeur en Occident depuis les Modernes, en rapport avec le développement de la science. 
arthritique (GLM, 1935) ${ }^{25}$, photo identitaire complémentaire de la notice du Dictionnaire abrégé du surréalisme : "PRASSINOS (Gisèle), née en 1920. - "Alice II." Poète surréaliste. (La Sauterelle arthritique (1934), Quand le bruit travaille, etc. $)^{26}$. » En voici brièvement les circonstances : le grand frère Mario Prassinos, plus âgé de quatre ans, jeune peintre qui fréquente alors le groupe surréaliste, découvre des textes écrits par sa sœur pour s'amuser dans le contexte d'un compagnonnage de création qui permet à la petite fille de partager avec les hommes de la famille (le père prestigieux, ancien professeur de littérature française et directeur de revue, et le frère), un domaine, celui de l'esprit, qui ne revient pas de droit aux femmes dans cette famille grecque d'origine orientale. André Breton accueille ces textes avec enthousiasme : il y voit la preuve de l'existence de la poésie spontanée. Gisèle Prassinos incarne aussitôt la « figuration prophétique [...] accomplie $^{27} \gg$ de l'allégorie de l'écriture automatique illustrant la couverture de La Révolution surréaliste de 1927.

L'apparente transparence de cette révélation, au propre et au figuré, par la photo, est ce qui pose question à l'avant-gardisme du mouvement : l'autre façon d'être au monde qu'elle tente de cautionner n'est-elle pas travaillée par une problématique du genre qui ferait de la femme son point aveugle et expliquerait in fine la place des femmes dans le mouvement surréaliste? Nous traiterons cette question en deux temps : de quelle ontologie nouvelle procède donc la surréalité de la femme-enfant? Et ce «mythologème » $\mathrm{y}$ a-t-il véritablement sa place ou vient-il en troubler, voire en contrecarrer la portée avant-gardiste?

« J'illustrais leur théorie ${ }^{28}$ », dira plus tard Gisèle Prassinos. La mise en scène d'une révélation - photographique et événementielle - est évidente, confirmée avec humour par Gisèle Prassinos dans un récit où elle note combien il avait été difficile de " prendre des attitudes très naturelles ${ }^{29}$ ». D'ailleurs une première prise du même moment existe, différente ${ }^{30}$.

Le sujet de la photo est évidemment le texte que lit, les yeux baissés, une petite fille sous le regard aimanté de voyants tournés vers elle jusqu'à la fascination, visible chez André Breton assis au premier plan avec Éluard et situé à l'extrême gauche, en pendant de Gisèle Prassinos à l'extrême droite.

25. La photo de Man Ray figure dans l'exposition au Centre Pompidou « La Subversion des images. Surréalisme, photographie, film » (novembre 2010-janvier 2011).

26. André Breton et Paul Éluard, Dictionnaire abrégé du surréalisme [1938], repris dans André Breton, Euvres complètes, t. II, op. cit., p. 835.

27. Gérard Legrand, op. cit., p. 10.

28. Cité dans Annie Richard, Le Monde suspendu de Gisèle Prassinos, op. cit., p. 26.

29. Gisèle Prassinos, Avant-Propos de Le Rêve, Paris, Éditions de la revue Fontaine, coll. «L'âge d'Or », 1947, p. 12.

30. La photo a été montrée lors de l'exposition « Le Monde suspendu de Gisèle Prassinos ». 
Au second plan, debout, de gauche à droite, Mario, le frère, son ami Henri Parisot, Benjamin Péret et le grand René Char qui domine la " petite », un bras appuyé au-dessus d'elle. Moment surréel par excellence d'une assemblée notoire de poètes frappée de stupeur devant un phénomène qui ne peut à son tour que plonger le regardeur de la photo dans un étonnement profond. Le médium photographique, nous le savons, est privilégié par les surréalistes à cause de la technicité de l'enregistrement du réel similaire à l'automatisme recherché de l'écriture, définie par André Breton comme « véritable photographie de la pensée ${ }^{31} \gg$. La photographie, étymologiquement écriture de la lumière, donne à voir un au-delà du visible : une théorie dans le sens premier de contemplation, de vision abstraite, de spéculation, en l'occurrence une des « épiphanies tangibles de la femme-enfant ${ }^{32}$ » garante de la réalité de l'écriture automatique. Cette photo-document, photo-manifeste, les textes se chargeront largement de l'expliciter ${ }^{33}$, mais nous faisons le choix d'approcher la dimension ontologique de l'image en elle-même : que cherche-t-elle à objectiver, quel schème de qualités du réel rend-elle perceptible?

Un contraste est visible entre les attitudes individualisées des hommes et celle de la liseuse tout entière absorbée, dissoute dans sa lecture : chacun se tient d'une façon particulière qui dénote une réaction personnelle, intérieure devant le phénomène dont il est témoin. L'expression d'André Breton est sans doute la plus significative de l'étonnement, chef de groupe oblige. Certes Gisèle Prassinos, dans son rôle de lectrice, n'est pas appelée à montrer ce qui l'anime : néanmoins la comparaison avec la représentation allégorique à laquelle elle renvoie, la photo de «l'écolière ambiguë ${ }^{34}$ », montre une différence notable. On se souvient de cette " garçonne» haut perchée sur un tabouret écrivant les yeux tournés vers le côté opposé de son pupitre, vers un ailleurs d'où lui vient sans doute l'inspiration. De l'allégorie à la photo de Gisèle Prassinos, c'est tout le passage de l'idée à la réalité extérieure : la photo entre dans la stratégie d'André Breton ${ }^{35}$ pour réconcilier l'idéalisme des débuts et le matérialisme des années 1930. La photo de Gisèle Prassinos s'inscrit dans une période de crise de l'automatisme telle que l'exprime la citation bien connue : «L'histoire de l'écriture automatique dans le surréalisme serait, je ne crains pas de le dire, celle d'une infortune continue $^{36}$. $\gg$ C'est le temps de la production théorique de l'objectivation,

31. André Breton, «Max Ernst» [1921], Les Pas perdus [1924], repris dans Euvres complètes, t. I, op. cit., p. 245.

32. Gérard Legrand, op. cit., p. 100.

33. Notamment La Sauterelle arthritique, op.cit., préfacé par Paul Éluard.

34. Voir La Révolution surréaliste, op. cit. et l'entrée « Automatique (écriture) », Dictionnaire abrégé du surréalisme, op. cit., p. 791.

35. Michel Poivert, «Politique de l'éclair: $\mathrm{AB}$ et la photographie », Études photographiques, $\mathrm{n}^{\circ}$ 7, 2000 [En ligne], http://etudesphotographiques.revues.org/index207.html.

36. André Breton, «Le message automatique» [Minotaure, $\left.n^{\circ} 3-4,1933\right]$, Point du jour [1934], repris dans Euvres complètes, t. II, op. cit., p. 380. 
qui vise à redéfinir l'automatisme sous l'éclairage de l'adhésion au matérialisme historique et à avancer sur le chemin tracé dès le départ, fondement de l'avant-gardisme du mouvement, à « exprimer, soit verbalement, soit par écrit, soit de toute autre manière, le fonctionnement réel de la pensée ${ }^{37}$ ».

Or la science «présente dans l'imaginaire surréaliste sous forme métaphorique tout au long des années 1920 est désormais appelée à remplir un rôle plus précis : celui d'une légitimation des théories surréalistes face à la critique $^{38} \gg$. André Breton accordait un grand prix à l'ouvrage de Bachelard Le Nouvel Esprit scientifique (1934), et à celui d'Henri Poincaré, La Valeur de la science (1906), qu'il recommande à Jacques Doucet pour son projet de bibliothèque. Il se représente lui-même parodiquement en savant dans le photomontage L'Écriture automatique qui accompagne sa propre notice dans le Dictionnaire abrégé du surréalisme : le visage de Breton étudiant est accolé à un corps étranger, tandis qu'une femme est présente en fond derrière une vitre, « la femme en cage qui attend son tour, jouant sur le registre humoristique la femme cobaye des expériences menées par Breton ${ }^{39} »$; on perçoit sous le microscope des formes apparentées à l'image associée à l'écriture automatique, c'est-à-dire celle d'un tracé lumineux évoquant et produisant son objet.

L'objectif photographique senti comme « œil », « rétine artificielle $»^{40}$, capte le surgissement de l'être dans la ligne des photos spirites qui tentent à la fin du XIX siècle de fixer sur la plaque « les émanations de la pensée, les rêves, les images mentales ${ }^{41}$ ». Ainsi le 27 mai 1896, à la suite de la découverte des rayons X en 1895 par Wilhlem Conrad Rôntgen, le commandant Louis Darget fait « la photographie fluidique de la pensée » en posant la main sur son front, main en contact avec une plaque photo, puis dans la même série " photo fluidique de la pensée », il place une plaque sur le front de sa femme endormie et obtient la « photographie du rêve, 25 juin $1896 »^{42}$. La photo est le processus même de l'éclair de la pensée consciente que Breton déplace avec la psychanalyse à la pensée inconsciente dans un « surrationalisme » qu'il emprunte à Bachelard ${ }^{43}$.

37. André Breton, Manifeste du surréalisme [1924], repris dans Euvres complètes, t. II, op. cit., p. 328.

38. Michel Poivert, «Politique de l'éclair : AB et la photographie », op. cit., p. 2.

39. Ibid.

40. Monique Sicard, « La "photo-graphie" entre nature et artefact », dans Philippe Descola (dir.), La Fabrique des images, op. cit., p. 118.

41. Savine Faupin, «Dessin animé. Spiritisme, automatisme, métamorphoses », dans Hypnos, images et inconscient en Europe, catalogue de l'exposition, Lille, Musée d'Art Moderne, 14 mars-12 juillet 2009, Lille, Musée d'Art Moderne Lille Métropole, 2009, p. 50. 42. Ibid.

43. Voir Gaston Bachelard, «Le surrationalisme» [Inquisitions, $n^{\circ}$ 1, juin 1936], L'Engagement rationaliste, Paris, PUF, 1972, p. 7-8, et André Breton, «Crise de l'objet » [Cahiers d'Art, $\left.\mathrm{n}^{\circ} 1-2,1936\right]$, Le Surréalisme et la peinture [1928; 1965], repris dans Euvres complètes, t. IV, Paris, Gallimard, coll. « Bibliothèque de la Pléiade », 2008, p. 681-688. 
C'est dans l'esprit du «statut d'instrument d'accès à l'objectivité qui fut alors conféré à la nouvelle image ${ }^{44} »$ que s'inscrit la photo de la rencontre de Gisèle Prassinos et du groupe surréaliste en 1934 : l'introduction du regard observateur collectif, qui est la grande différence par rapport à la photo allégorique de 1927, et sa mise en scène entre la première et la deuxième prises, relèvent d'une ontologie naturaliste, voire surnaturaliste. La photo est naturaliste par le regard à la fois collectif et différencié de sujets face à un phénomène. Elle est le médium privilégié du " monde objectif ${ }^{45}$ " qui naît sous l'impulsion de la science moderne aux alentours du XVII siècle en Europe, monde matériel qui inclut par ses caractères physiques l'être qui le contemple, mais s'en différencie par l'intériorité exclusivement humaine de la conscience. Le Centre de recherches sur le surréalisme ${ }^{46} \mathrm{~s}$ 'interroge sur les rapports du surréalisme et du baroque concomitant à l'émergence de l'astronomie : selon Jean-Claude Vuillemin ${ }^{47}$, la lunette astronomique pointée vers le monde depuis Copernic ruine la dichotomie entre un monde

44. Monique Sicard, op. cit., p. 113.

45. Philippe Descola définit les visions du monde selon quatre manières de saisir et de figurer des « continuités ou discontinuités que les humains identifient entre eux et le reste des existants-congénères, organismes ou artefacts sur le double plan physique et moral », synthétisées dans le cartel introductif de l'exposition par :

- un «monde animé » pour l'animisme avec des « ressemblances morales et différences physiques » (animaux et plantes ont une " âme » et sont perçus comme ayant une intériorité semblable à celle des humains);

- un "monde subdivisé » avec des "ressemblances morales et continuités physiques » pour le totémisme (recours à un ancêtre commun, certaines espèces d'animaux ou plantes pris comme modèles de même essence organique d'un groupe humain);

- un "monde enchevêtré » pour l'analogisme dominant en Europe de l'Antiquité à la Renaissance ainsi qu'en Extrême Orient et chez les Indiens d'Amérique (reposant sur la faculté d'établir des correspondances entre les êtres animés ou non, perçus comme tous différents, singuliers, moralement et physiquement; ce monde s'organise à partir d'analogies entre notamment microcosme, l'univers, et macrocosme, la personne humaine, ou entre des catégories comme le masculin correspondant par exemple au sec opposé au féminin assimilé à l'humide; la pensée analogique constitue aisément des réseaux comme chez les Indiens Hopi où chaque esprit est une qualité du monde ou des associations dont une figure typique est la chimère une et composite);

- un «monde objectif» pour le naturalisme caractérisé par des différences morales sur fond de continuité physique (l'intériorité humaine est unique face à la Nature, prise au sens large, de totalité des existants, notion centrale qui englobe les humains pour les caractères physiques mais les humains sont les seuls à avoir une conscience, un esprit; pour les Modernes, parallèlement au développement de la science, grandit ainsi l'espace autonome d'un sujet au sein d'un monde physique qui s'affranchit du religieux) (Philippe Descola, " Manière de voir, manières de figurer ", dans La Fabrique des images, op. cit., p. 13).

46. Centre de recherches sur le surréalisme dirigé par Henri Béhar, Université Paris 3 Sorbonne nouvelle. Séminaire 2010-2011 : « Surréalisme et Baroque ».

47. Lors de la séance du 5 novembre 2010 : «Baroque : un concept surréaliste? Débat sur le baroque surréaliste ». Voir aussi Diana Vlasie et Jean-Claude Vuillemin, « Theatrum mundi : désenchantement et appropriation », Poétique, nº 158, 2009, p. 173-199. 
engendré intelligible et un monde sublunaire hors d'atteinte qui formait un univers clos et ordonné, gouverné par les analogies. Le regard libéré scrute désormais les lois mystérieuses et invisibles qui régissent une réalité à décrypter, sujette à l'illusion d'optique, invitant l'œil à se décentrer pour saisir le caché comme dans l'anamorphose ou dans la recherche de l'ambiguïté des formes, si fréquente dans la peinture surréaliste ${ }^{48}$.

La femme-enfant s'insère parfaitement dans ce puzzle épistémologique comme notion issue du discours scientifique freudien, dans la ligne d'un savoir médical constitué à partir des études sur l'hystérie de Charcot ${ }^{49}$. Fritz Wittels, psychanalyste né à Vienne, contemporain et disciple de Freud, redécouvert par Edward Timms ${ }^{50}$, est un des membres de la société psychanalytique de Vienne et le premier biographe de Freud. Ses Mémoires, écrits à New York dans les années 1940 et relégués dans l'ombre, « révèlent que durant la première décennie $\mathrm{du} \mathrm{xx}^{\mathrm{e}}$ siècle, les recherches de la Société psychanalytique de Vienne portèrent directement sur le demi-monde viennois dont, celle, et non la moindre, relative aux cultes érotiques discutables qui entouraient la "femme-enfant", Irma Karczewska ${ }^{51}$ ». « La femme-enfant » est le titre d'un article de Wittels lu à Freud en privé et présenté à la Société psychanalytique puis publié dans le magazine de Karl Kraus, Le Flambeau: Wittels dit utiliser le cadre freudien de l'enfance "perverse et polymorphe " développé dans Trois essais sur la théorie sexuelle (1905). «Elle avait été "perverse polymorphe" comme l'est un petit enfant et n'avait jamais développé d'inhibitions culturelles d'aucune sorte $^{52}$.»

Quid dans ces conditions de la "surréalité 》 avant-gardiste de la photo? Elle est évidemment du côté de ce que suggère le regard enchanté des témoins : une dimension inconnue et vertigineuse de la réalité que seul « le sens mythique», comptable de l'étrangeté de soi et du monde, peut toucher : le «mythologème » nouveau de la femme-enfant fait office de pensée 《 figurative ${ }^{53}$ affranchie du primat de la raison et du positivisme, à la charnière du conscient et de l'inconscient, fusionnant sujet et objet. À cet égard, la pose étonnée des voyants met en abyme l'éclair de la prise mécanique. La photo-objet érige une simple séance de lecture en credo : le sujet collectif, capable de discernement, celui des discours, en est

48. Exposition « Une image peut en cacher une autre » (Grand Palais, 8 avril 2009-6 juillet 2009), avec notamment le tableau de Man Ray au titre significatif Le Rébus (1938).

49. Jean-Martin Charcot, illustre médecin français de la fin du XIX ${ }^{\mathrm{e}}$ siècle, professeur réputé à l'hôpital de La Salpêtrière à Paris dont Freud vient suivre les cours en 1885.

50. Voir Freud et la femme-enfant, Mémoires de Fritz Wittels, Paris, PUF, coll. « Bibliothèque de la psychanalyse », 1999, page de garde.

51. Ibid., p. IX.

52. Ibid., p. 70.

53. Louis Aragon, Le Paysan de Paris [1926], Paris, Gallimard, coll. « Folio », 1972, respectivement p. 155 et p. 143. 
essentiellement masculin, de même que le phénomène de l'écriture automatique, dans sa naïveté, est essentiellement féminin.

L'ontologie naturaliste ou surnaturaliste de la photo de la femmeenfant rend ainsi «tangible » la structure fondamentalement binaire des sexes qui la sous-tend, mais ce mythe a-t-il de ce fait réellement sa place dans le projet de bouleversement anthropologique avant-gardiste d'une saisie profonde, totale du réel?

De quel réel s'agit-il en effet sur la photo? La problématique du genre qui dénaturalise la différence des sexes permet de voir le décalage épistémologique entre l'objectif recherché et le médium utilisé, à savoir l'image de la femme-enfant.

Si les sujets masculins peuvent à travers un être féminin de chair et d'os contempler l'entité de l'écriture automatique auquel il est censé renvoyer, c'est qu'ils le perçoivent sur un mode différent, voire antinomique de celui qu'ils recherchent : le mode analogique à l'œuvre dans le genre. La femmeenfant entre visiblement dans le système symbolique d'oppositions structuré par la dichotomie féminin-masculin, tel que l'a conceptualisé notamment l'anthropologue Françoise Héritier, qui désigne la construction culturelle du genre à partir de l' " observation liminaire de l'étonnante et fondamentale différence sexuée ${ }^{54}$ » comme la base de nos machines mentales binaires, variables dans une certaine mesure selon les cultures.

Que la femme soit dans le surréalisme le médium sacré, non individualisé, de l'univers, contrairement à l'homme, sujet agissant et autonome, correspond à une cosmologie traditionnelle inséparable de pratiques sociales, le tandem nature/culture associé au rapport féminin/masculin. L'anthropologue Colette Guillaumin écrit que si les femmes sont considérées comme plus naturelles que les hommes, c'est parce qu'elles sont constituées en choses ${ }^{55}$ : le regard de l'assemblée masculine sur la photo désigne en Gisèle Prassinos un objet d'étude, en l'occurrence le produit brut de la nature humaine à l'état doublement primitif de femme et d'enfant.

Il est aisé de superposer à cette image celle du célèbre tableau d'André Brouillet Une leçon clinique à La Salpêtrière ${ }^{56}$. La psychanalyse prend le relais de cette approche analogique avec la notion de « continent noir », de développement psychique du sujet féminin qui reste en deçà du symbolique, aboutissant au fameux « la femme n'existe pas » de Lacan : la «femmeenfant » procède d'un des grands récits, au sens de Lyotard, en l'occurrence

54. Françoise Héritier, Masculin/Féminin II : dissoudre la hiérarchie, Paris, Odile Jacob, 2002, p. 15.

55. Colette Guillaumin, Sexe, race et pratique du pouvoir, Paris, Côté-Femmes, 1992, p. 49. 56. Le tableau, exposé au Salon des indépendants de 1887, montre, lors d'une leçon de Charcot, la patiente hystérique Blanche Wittmann à un public exclusivement masculin principalement constitué d'étudiants et de médecins. 
de la construction culturelle du genre féminin du côté de l'absence de maîtrise et de la nature, par opposition au masculin porteur de la civilisation $^{57}$. La femme-enfant est un avatar de Salomé, innocente perverse en vogue particulièrement à la fin $\mathrm{du} \mathrm{XIX}^{\mathrm{e}}$ siècle et sujet de fascination de Breton contemplant les tableaux de Gustave Moreau ${ }^{58}$.

Le discours surréaliste, profondément genré à son insu, confond, comme le fait l'époque et comme les recherches actuelles permettent de le décrypter, le sexe et le genre tel qu'il a été institué par toute une culture. Le genre, point aveugle de la théorie avant-gardiste du surréalisme, conforte finalement dans le mythe de la femme-enfant une figure typique de l'ontologie analogiste traditionnelle : le mirage de la chimère ${ }^{59}$. La femme-enfant est un être hybride, somme en effet de deux réalités fondamentalement hétérogènes, l'une procédant de l'autre depuis la nuit des temps : la femme à l'enfant renvoyant aux sources de l'humanité laisse place à la femme et l'enfant réunis en une entité unique monstrueuse dont le nom composé à lui seul fonde la fiction visuelle.

Car la question se pose, comme pour toute chimère, de sa représentation : sur la photo de Man Ray, c'est plus la dénomination qui instaure et perpétue dans la réception l'évocation de la femme-enfant que l'image elle-même de cette petite fille de quatorze ans, endimanchée pour la circonstance et intimidée par une assemblée admirative pour le moins insolite, surtout lorsque l'on connait la biographie de Gisèle Prassinos. Peu importe au groupe la personne de Gisèle Prassinos : la vision analogiste en fait le paradigme d'épiphanies réelles et imaginaires égrenées par André Breton dans sa notice de l'Anthologie de l'humour noir - la reine Mab, la « jeune chimère » de Max Ernst, « l'écolière ambiguë »- et par Gérard Legrand à sa suite ${ }^{60}$ : Bettina Von Arnim, Violette Nozières, la Juliette de Shakespeare célébrée par les romantiques allemands, la Poupée de Bellmer et la Mélusine d'Arcane 17.

Que la Gisèle Prassinos réelle s'efface pour la postérité derrière une idée, aux antipodes de l'objectivité, c'est ce que prouve encore une fois le hasard objectif, un article du Monde-Télévision du 12 avril 2003 présentant un film de Fabrice Maze, André Breton, malgré tout et illustré par la photo

57. Voir Monique Schneider, Généalogie du masculin, Paris, Aubier, 2000, et Le Paradigme féminin, Paris, Aubier/Flammarion, 2004.

58. Voir Annie Richard, "Salomé ou les avatars de la femme-enfant », dans La Femme s'entête, op. cit.

59. Voir « la "jeune chimère" de Max Ernst » qu'évoque Breton dans la notice de l'Anthologie de l'humour noir, op. cit., p. 1167. Il s'agit d'un collage gouache et encre sur papier de 1920 ou de l'huile de 1935 La Jeune Chimère en tenue de soirée, prémonitoire de la rencontre avec Leonora Carrington.

60. Voir Gérard Legrand, op. cit. 
de Man Ray dont la légende énumère le nom des hommes sans mentionner Gisèle Prassinos devenue « accessoire, transparente, sans nom ${ }^{61}$ ».

Tout se passe finalement comme si le lien entre le principe de pensée subversif de l'avant-garde surréaliste, l'écriture automatique et le genre essentialisé - la femme-enfant - tenait lieu de verrou à la structure cognitive archaïque issue de «l'observation liminaire de l'étonnante et fondamentale différence sexuée ${ }^{62} »$. Le voilà incarné, ce point sublime, par une chimère, aporie manifeste qui éclaire la présence-absence des femmes dans le surréalisme et tout autant la difficulté pour avancer dans le domaine de la pensée si on ne se dégage pas du substrat différentialiste des sexes : celui-ci a été établi dans notre culture et inscrit dans la langue par une épistémologie analogiste contradictoire à l'épistémologie d'invention - au sens de rencontrer, invenere - du monde par un sujet agent de la pensée. Le sujet masculin pris en relation avec le féminin dans une catégorisation dualiste figée est libéré tout autant que le sujet féminin par l'approche constructiviste du genre. La photo en est là encore la preuve/épreuve : le spectateur contemporain, engagé sur la voie du sujet personnel, interroge à son tour la scène mentale exhibée par la photo. Mais il lui suffit, pour y voir autre chose qu'une figure féminine attendue, de décentrer le regard en le focalisant non plus à partir du groupe, mais derrière les yeux fermés de la jeune poète, sujet à part entière qui n'aura de cesse de dépasser la barrière des sexes, confortée par son alter ego de création, son frère relais depuis toujours entre les mondes masculin et féminin clivés de sa famille orientale.

Monde? La plongée dans l'étymologie ramène une autre clé : monde vient d'émonder, c'est-à-dire rendre propre, débarrasser du superflu; le mot indique comment s'appréhende le réel, par un geste de tri et de façonnement dont l'équivalent dans le discours est la capacité discriminante de l'identique et du différent à l'œuvre dans le langage. La remettre en cause était le mot d'ordre du surréalisme pour assurer l' « essor de la pensée » : le genre, qui met au jour ce processus à la base même de la réalité humaine du partage des sexes, est l'outil contemporain avant-gardiste par excellence, capable de satisfaire à la grande ambition surréaliste de redonner à l'esprit l'accès aux choses.

Annie Richard

61. Annie Richard, "Pour Gisèle Prassinos », Courrier des lecteurs, dans Le Monde, 19 avril 2003.

62. Françoise Héritier, op. cit., p. 19-20. 